\title{
Valor y distribución en Adam Smith
}

\author{
Roberto Rivera Campos
}

Se ha argumentado tradicionalmente que Adam Smith desarrolló dos teorias contradictorias del valor de cambio. Una de ellas en la cual Smith habría argumentado que el valor de las mercancias estaria determinado por la cantidad de trabajo empleado en su producción o adquisición y que tendria vigencia unicamente en el estado primitivo y rudo de la sociedad; y la otra, en la cual Smith argumentaria que el valor de cambio está determinado por la cantidad de trabajo que una mercancía puede adquirir, esta última noción tomaria su vigencia en el estado moderno de la sociedad, una vez que se ha acumulado capital y que la tierra ha sido apropiada. De acuerdo a esta interpretación, en la primera noción de valor de cambio habria un planteamiento en germen de lo que es la substancia del valor, es decir se estaria argumentando que la substancia del valor es la cantidad de trabajo incorporada en las mercancias y que la falta de desarrollo de las condiciones hístoricas le habria impedido a Smith llevar adelante esta idea.

En la primera parte de este capitulo se argumentará que en LA RIQUEZA DE LAS NACIONES hay una unica teoría del valor y en la segunda se estudiará la teoría de la determinación del ingreso y se argumentará que en un contexto dinámico la determinación de la tasa natural adquiere connotaciones especiales respecto a su determinación en un contexto estático. A lo largo del texto las citas de la Riqueza de las Naciones solamente se indicarán con el número de la página.

El presente trabajo es el resultado de los cursos que he estado impartiendo desde 1986 a los estudiantes del último afio de Economía en la Universidad Centroamericana José Simeón Cañas. Por supuesto 
mis reflexiones al respecto se han enriquecido notablemente por las discusiones que durante estos años hemos sostenido con las diferentes generaciones èn las aulas universitarias.

\section{VALOR DE CAMBIO Y TRABAJO.}

1. Antes de iniciar la elaboración de la teoria del valor, Smith define lo que entiende por valor de cambio de una mercancia como "la capacidad de comprar otros bienes, capacidad que se deriva de la posesión del dinero"(pag. 30), y establece que su objetivo será la investigación de los principios que regulan el valor de cambio de las mercancías. Para ello procurará poner en claro:

"Primero, cuál sea la medida de este valor de cambio, o en que consiste el precio real de todos los bienes. Segundo, cuáles son las diferentes partes integrantes de que se compone este precio real. Por ultimo, cuáles son las diferentes circunstancias que unas veces hacen subir y otras bajar algunas o todas las distintas partes componentes del precio, por encima o por debajo de su proporción natural o corriente"(pag.30).

2. El punto de partida de Smith consiste, entonces, en resolver el problema de la medida del valor de cambio. La importancia de escoger un patron de medida adecuado queda claro en el siguiente párrafo:

“..de la misma manera que una medida que estuviese siempre cambiando su longitud como el pie natural, el palmo o el brazo, no podria ser jamás una medida exacta de otras cosas, asi una mercaderia que varie continuamente en su valor, nunca podrá ser medida exacta de otros articulos."(pag. 33).

Para Smith ni el oro ni la plata constituyen medidas adecuadas para el valor de cambio de las mercancías:

"El oro y la plata, como cualquier otro bien, cambian de valor; unas veces son más caros, y otras, más baratos; unas veces son más fáciles, y otra más dificiles de adquirir. El descubrimiento de las ricas minas de América redujo el valor del oro y la plata, en el siglo XVI, a casi una tercera parte de su valor anterior"(pag. 33).

Smith procede entonces a plantearse al trabajo como la medida del valor de cambio. Para Smith trabajar representa un costo para el trabajador, pues siempre tiene que sacrificar a cambio del trabajar sus comodidades y su libertad. Podriamos interpretar lo anterior diciendo que el costo del trabajo para el trabajador es el grado de sacrificio de sus comodidades, de su libertad y de su felicidad por cada hora de 
trabajo realizado. Según Smith, este costo no cambia nunca:

"Iguales cantidades de trabajo, en todos tiempos y lugares, tienen, según se dice, el mismo valor para el trabajador. Presuponiendo un grado normal de salud, de fuerza y de temperamento, de aptitud y destreza, ha de sacrificar siempre la misma proporción de comodidad, de libertad y de felicidad. El precio que paga debe ser siempre el mismo, cualquiera que sea la cantidad de bienes que reciba en cambio."(pag. 33 y 35).

El trabajo es en consecuencia planteado como el patrón de medida del valor de cambio:

"Por consiguiente el trabajo, al no cambiar nunca de valor, es el unico y definitivo patrón efectivo, por el cual se compran y estiman los valores de todos los bienes, cualesquiera que sean las circunstancias de lugar y de tiempo."(pag. 34).

Habiendo establecido la invariabilidad del valor del trabajo, se puede entender que cuando por iguales cantidades de trabajo unas veces se obtiene mayor cantidad de bienes y otras menos, lo que está cambiando es el valor de las mercancias y no el del trabajo, aunque, como dice Smith, para quien emplea al trabajador le parezca que lo que se ha hecho más barato o más caro respectivamente sea el trabajo. Así, pues, Smith establece que el trabajo es el precio real de las mercancias y que la moneda sólo su precio nominal.

3. Hasta acá se ha argumentado que el trabajo es la medida correcta del valor y no ha sido necesario definir qué clase de trabajo. Todo lo que se ha dicho es que medido en trabajo se expresa el precio real de la mercancía.

De acuerdo a la definición original, el valor de cambio de una mercancia es su capacidad de comprar otros bienes. Como ya se estableció un patrón de medida para esta capacidad se puede avanzar y plantear conforme a Smith que:

"..el valor de cualquier bien, para la persona que lo posee y que no piense usarlo o consumirlo, sino cambiarlo por otros, es igual a la cantidad de trabajo que pueda adquirir o de que pueda disponer por mediación suya."(pag. 31).

De acuerdo a la definición anterior debe quedar claro que la cantidad de trabajo que mide el valor de las mercanclas es el que puede ser adquirido a cambio de la mercancia y no el empleado en su producción.

Para entender con mayor precisión esta definición y evitar errores de interpretación del texto del autor, se debe enfatizar que Smith define 
el valor de cambio de una mercancia a partir de su existencia en manos de su poseedor y de su disposición a cambiarla por otras mercancias. La misma definición se puede establecer desde dos angulos opuestos: desde el ángulo del poseedor, quien está dispuesto a desprenderse de ella a cambio de disponer de trabajo de otros; y desde el ángulo de su no poseedor, quien está dispuesto a padecer penas y fatigas, entregando trabajo a su actual poseedor a cambio de adquirir la mercancia en cuestión. Asi, afirma Smith:

"El precio real de cualquier cosa, lo que realmente le cuesta al hombre que quiere adquirirla, son las penas y fatigas que su adquisición supone. Lo que realmente vale para el que ya la ha adquirido y desea disponer de ella, o cambiarla por otros bienes, son las penas y fatigas de que lo librarán, y que podrá imponer a otros individuos."(pag. 31).

Si se tiene claridad del doble punto de vista se concluye que ambas formas de definir el valor de cambio son identicas. Desde la definición de Smith se entiende, consecuentemente, que el patrón de medida escogido para el valor de una mercancía es la cantidad de trabajo adquirible por medio de ella.

4. Una vez establecido que la medida del valor de cambio es la cantidad de trabajo adquirible(labour commanded), el problema siguiente para resolver es el de determinar cuáles son los factores que hacen que el valor de cambio de una mercancía sea mayor o sea menor en un momento determinado(sus reguladores), es decir, que hacen que pueda adquirir más o menos trabajo.

Para determinar las circunstancias que regulan el valor de cambio, Smith separa metodológicamente el análisis en dos etapas históricas: el estado primitivo y rudo de la sociedad, previo a la acumulación de capital y a la apropiación de la tierra; y el estado, que se podria denominar moderno, en el cual ya se ha dado la acumulación de capital y la tierra ya ha sido apropiada. Siguiendo a Smith se abordará el problema en el orden descrito.

4.1. Smith demuestra que en el estado primitivo y rudo de la sociedad la cantidad de trabajo adquirible por un bien, es decir, su valor de cambio, queda regulado por la cantidad de trabajo que se ha empleado en su producción o en su adquisición.

"En el estado primitivo y rudo de la sociedad, que precede a la acumulación de capital y a la apropiación de la tierra, la única circunstancia que puede servir de norma para el cambio reciproco de diversos objetos parece ser la proporción entre las distintas clases de trabajo que se necesitan para adquirirlos."(pag.47). 
Smith utiliza su conocido ejemplo del castor y el ciervo, argumentando que en las condiciones descritas, si cazar un castor cuesta el doble de trabajo que cazar un ciervo, será natural que un castor se intercambie por dos ciervos. La razón de que esto ocurra asi la da en los siguientes términos:

"En ese estado de cosas el producto integro del trabajo pertenece al trabajador, y ( por consiguiente) la cantidad de trabajo comunmente empleado en adquirir o producir una mercancia es la única circunstancia que puede regular la cantidad de trabajo ajeno que con ella se puede adquirir, permutar o disponer."

De acuerdo a lo que se ha desarrollado hasta acá, en este estadio de la sociedad el valor de las mercancias se mide por el trabajo adquirible y debido a las condiciones históricas de existencia de esta sociedad, solamente hay un regulador para el valor de cambio, el trabajo empleado o gastado. Resulta entonces que aqui el trabajo adquirible y el trabajo empleado coinciden cuantitativamente, pero sigue siendo el primero el patrón de medida del valor. Por ser el único regulador del valor de cambio, si el trabajo empleado se duplica el trabajo adquirible también se duplicará y, por lo tanto, también lo hará el valor de cambio.

Jean Cartelier sostiene la misma tesis que acá estamos desarrollando, a saber: que en Smith, aún en el estado rudo y primitivo de la sociedad el valor se mide por la cantidad de trabajo adquirible, y que la cantidad de trabajo empleado sólo es el regulador de la cantidad de trabajo adquirible:

"Es decisivo observar que la cantidad de trabajo incorporado (empleado, en nuestros términos) no es el valor, pero determina la cantidad de valor, que es una cantidad de trabajo exigido (adquirible, en nuestros términos). En otras palabras, aún en el estado primitivo y rústico, el valor queda definido por una relación de intercambio con el trabajo. En cambio, la cantidad de trabajo incorporado desempeña un papel de determinación cuantitativa: es la cantidad de trabajo incorporado la que determina la amplitud del trabajo exigido"(Cartelier, pag, 179).

Sin embargo, Cartelier no deja de ver algunas contradicciones en las afirmaciones de Smith. En el párrafo siguiente de Smith:

"El precio real de cualquier cosa, lo que realmente le cuesta al hombre que quiere adquirirla, son las penas y fatigas que su adquisición supone."(pag. 31).

Según Cartelier, aqui Smith estaria sugiriendo que el trabajo aparece como la fuente o la sustancia del valor. Desde el doble punto de 
vista que hemos definido arriba para entender el establecimiento de la medición del valor de Smith, el punto de vista del poseedor, que desea disponer de ella, y el punto de vista de su no poseedor, que desea adquirirla, no habría ninguna contradicción, pues en la segunda, que es la que Smith está usando acá, se trata siempre del trabajo adquirible por el poseedor de la mercancia.

Más duda surge en el siguiente párrafo de Smith:

"Lo que se compra con dinero o con otros bienes, se adquiere con el trabajo, lo mismo que lo que adquirimos con el esfuerzo de nuestro cuerpo. El dinero o sea otra clase de bienes nos dispensan de esa fatiga. Contienen (el dinero u otros bienes de los cuales pensamos disponer) el valor de una cierta cantidad de trabajo, que nosotros cambiamos por las cosas que suponemos encierran, en un momento determinado, la misma cantidad de trabajo."(pag. 31).

Según Cartelier, la confusión que aquí surge es total. Teniendo presente el orden global en que el autor ha ido estableciendo la medición del valor, la confusión comienza a disminuir. Ciertamente las palabras "contienen" y "encierran" llevan a la confusión, pero ellas aparecen aisladas en el argumento total de Smith que está dirigido a determinar la medición del valor y no su contenido.

4.2. Cuando las condiciones historicas de la sociedad cambian en el sentido antes indicado, surgen otros reguladores del valor de cambio adicionales al trabajo empleado. Smith argumenta en dos partes:

"Más tan pronto como el capital se acumula en poder de personas determinadas, algunas de ellas procuran regularmente emplearlo en dar trabajo a gentes laboriosas, suministrandoles materiales y alimentos, para sacar un provecho de la venta de su producto o del valor que el trabajo incorpora a los materiales. Al cambiar un producto acabado, bien sea por dinero, bien por trabajo, o por otras mercaderias, además de lo que sea suficiente para pagar el valor de los materiales y los salarios de los obreros, es necesario que se dé algo por razón de las ganancias que corresponden al empresario, el cual compromete su capital en esa contingencia."(pag. 48).

En consecuencia concluye:

"La cantidad de trabajo que se gasta comunmente en adquirir o producir una mercancia no es la única circunstancia que regula la cantidad susceptible de adquirirse con ella, permutarse o cambiarse. Evidentemente hay una cantidad adicional que corresponde a los beneficios del capital".(pag.49). 


\section{$Y$ agrega:}

"Desde el momento en que las tierras de un pais se convierten en propiedad privada de los terratenientes, éstos como los demás hombres, desean cosechar donde nunca sembraron, y exigen una renta hasta por el producto natural del suelo... Ha de pagar (el trabajador) al terrateniente una parte de lo que su trabajo produce o recolecta. Esta porción, o lo que es lo mismo, el precio de ella, constituye la renta de la tierra, y se halla en el precio de la mayor parte de los artículos como un tercer componente."(pag. 49).

En la cita 3 de la página 49 se lee que en la primera edición de la obra, Smith afirmaba:

En tal situación, ni la cantidad de trabajo habitualmente empleada en "adquirir o producir un artículo, ni las utilidades del capital que anticipó los salarios y suministró los materiales del trabajo, son las únicas circunstancias susceptibles de regular la cantidad de trabajo que comunmente debe adquirirse o de que en último término se dispone, para fines de intercambio. Una tercera circunstancia debe tenerse en cuenta, igualmente: la renta de la tierra."

El valor real, pues, de todas las mercancias está constituido por la adición de tres elementos: el trabajo gastado, el beneficio y la renta. Según varie cada uno de estos componentes, asi variará el valor de cambio de la mercancia. Mientras que en el estado rudo y primitivo de la sociedad el trabajo gastado era el único regulador de la cantidad de trabajo adquirible, del valor de cambio, en la sociedad moderna participan de igual función el beneficio y la renta.

Un ejemplo numérico servirá para afianzar estas ideas.Si se supone que empleando 10 horas de trabajo se pueden producir 100 unidades de una mercancia que consumen los trabajadores se pueden presentar los siguientes casos:

a. No se ha establecido la acumulación de capital, ni se ha apropiado la tierra, en consecuencia, todo el producto del trabajo pertenece al trabajador. Las cien unidades se las apropiará el trabajador. Si acaso se quisieran intercambiar estas cien unidades por trabajo se podría adquirir en condiciones normales ni más ni menos que 10 horasde trabajo ajeno, en consecuencia el precio real de estas 100 unidades seria 10 horas de trabajo, o lo que es lo mismo, cada unidad valdria $1 / 10$ de hora de trabajo. Como se aprecia, el trabajo adquirible coincide cuantitativamente con el trabajo empleado. Si en lugar de emplear 10 horas de trabajo para producir estas 100 unidades hubiese sido necesario emplear 8 horas, su valor de cambio, o lo que es to 
mismo el trabajo adquirible, habria disminuido a 8 horas de trabajo. Por esta razón se dice que el trabajo empleado es el único regulador del valor de cambio.

En este estado de cosas:

trabajo adquirible (igual) trabajo empleado

b. Ya se estableció la acumulación de capital y el capitalista adelanta los salarios del trabajo por lo cual reclama un beneficio, el cual se supone que asciende a 20 unidades. En estas condiciones al trabajo corresponden únicamente 80 unidades, lo cual significa que entregando a los trabajadores en forma de salarios 80 unidades, el capitalista puede disponer de otras 10 horas de trabajo, es decir, por cada unidad producida se podrá adquirir 1/8 de hora de trabajo, en consecuencia , medido en trabajo adquirible, el precio de cada unidad será $1 / 8$ de hora de trabajo, resultando en que el valor de cambio de las 100 unidades, es decir la cantidad de trabajo adquirible, se elevará a 12.5 horas. En estas circunstancias, mientras que la cantidad de trabajo empleado en producir las mercancias tue 10 horas, la cantidad adquirible por ellas es 12.5 horas. Las 10 horas de trabajo empleado bajo la dirección capitalista, dieron como resultado un producto que vale 12.5 horas de trabajo, del cual los trabajadores recibirán un valor de 10 horas, y los capitalistas se apropiarán de 2.5 horas, la tasa de ganancia será, en consecuencia, $25 \%$. Si acaso el beneficio ascendiera a 50 unidades, el valor de cambio de las 100 unidades producidas ascenderia a 20 horas de trabajo. Asi, pues, el beneficio se convierte en un segundo regulador del valor de cambio. En general, si el baneficio es mayor que cero, se dará la siguiente relación:

trabajo adquirible (mayor que) trabajo empleado

Con la noción de trabajo adquirible Smith ha logrado demostrar que los trabajadores, quienes emplearon 10 horas de trabajo, recibirán una remuneracion equivalente a 10 horas de trabajo adquirible, por medio de la cual si acaso desean disponer del dinero o de las mercancias recibidas, podrian obtener un trabajo por un valor de 10 horas, es decir podrian liberarse de las penas y tatigas que 10 horas de trabajo suponen. En consecuencia, conforme a esta argumentación, los trabajadores reciben exactamente un valor equivalente a su trabajo; la ganancia, pues, no resulta de la apropiación de un trabajo no pagado a los trabajadores, sino que es la recompensa que recibe el capitalista por haber comprometido el capital en tal contingencia. Se ha argumentado algunas veces que Smith estuvo cerca de haberse planteado el problema de la plusvalia y en consecuencia de la explotación. En 
verdad Smith estuvo muy lejos de plantearse ese problema, por el contrario, como acabamos de argumentar, todo su razonamiento estuvo orientado a demostrar el origen no explotador de las ganancias.

5. De acuerdo a lo anterior, el valor de cambio está regulado por la cantidad de trabajo empleado, el beneficio y la renta. Smith cierra su teoria afirmando que a su vez cada uno de estos elementos se mide por la cantidad de trabajo que cada uno de ellos puede adquirir:

"El valor real de todas las diferentes partes que componen el precio se mide, según podemos observar, por la cantidad de trabajo que cada una de esas porciones dispone o adquiere. El trabajo no sólo mide el valor de aquella parte del precio que se resuelva en trabajo, sino tambien el de aquella otra que se traduce en renta y en beneficio."(pag. 49).

Smith sale al paso a la posible crítica que podria formularsele a su teoria de que no toma en cuenta un cuarto elemento, a saber: el valor de los materiales de trabajo, argumentando que estos a su vez han sido productos de un trabajo anterior y que por lo tanto su valor tambien se resuelve en trabajo, beneficio y renta. Analizando el precio del grano Smith concluye:

"Asi pues, aunque el precio del grano pague el precio del animal y su mantenimiento, la suma total se descompondrá inmediata o finalmente en los tres componentes de siempre: renta trabajo y beneficio."(pag. 50).

6. Del análisis microeconómico del precio pasa directamente al macroeconómico del valor anual del producto nacional:

"Así como el precio o valor en cambio de cada mercancia en particular, y tomada separadamente, se resuelve en una o en otra de estas tres partes, o bien en todas ellas, de igual suerte el de todas las mercancías que componen el valor anual del producto de cada nación, considerado en su conjunto, se reduce necesariamente a esas tres porciones, y se distribuye entre los diferentes habitantes del pais como salarios de su trabajo, beneficios de su capital o renta de su tierra...Salarios, beneficio y renta son las tres fuentes originarias de toda clase de renta y de todo valor de cambio. Cualquier otra clase de renta se deriva, en última instancia, de una de estas tres."(pags. 51,52).

La conclusión macroeconómica del valor costituye el punto de partida para el desarrollo de dos teorías: la primera, la fuente del crecimiento económico; y la segunda, la teoría de la distribución del ingreso. 
7. Smith argumenta que la economía de un pais puede crecer debido a que siempre es posible emplear más trabajo el presente año que el anterior debido a que en un pais civilizado en la mayoria de las mercancias entran en bastante proporción la renta y el beneficio:

"de donde resulta que el producto anual de su trabajo es siempre suficiente para comprar o disponer de una mayor cantidad de trabajo del que se emplea en obtener, manufacturar y transportar el producto al mercado. Si la sociedad se hallase en condiciones de emplear anualmente todo el trabajo del que puede disponer en el curso del año, como la cantidad de trabajo se incrementaría grandemente de uno a otro, el producto de cada uno de los años sucesivos se incrementaria de una manera enorme con relación al anterior."

La teoria de la formación del precio de las mercancías por la adición de tres componentes ( la teoría de la suma como la llamó Sraffa) que mostró que en una sociedad moderna el trabajo adquirible es siempre superior al trabajo empleado, permite que a nivel macroeconómico, Smith vea la lógica del crecimiento económico. Antes de abandonar este tema del crecimiento económico, el cual no es el objetivo de este trabajo, se puede analizar brevemente dos implicaciones del planteamiento anterior.

7.1. Smith desarrolla su argumento en contra de la clase de los rentistas (aquellos que además quieren cosechar donde nunca sembraron), de los ociosos:

"Pero no hay un sólo país en que el producto anual integro se emplee en mantener a los trabajadores. Los ociosos consumen en todos esos paises una gran parte del producto $y$, según sean las proporciones como se distribuye éste, anualmente, entre esas dos clases tan opuestas, asi crecerá, disminuirá o permanecerá estacionario cada año su valor promedio o corriente."(pag. 53).

Este es el primer lugar en que Smith deriva directamente de su teoría del valor una consecuencia para la lucha de clases. Es evidente en el argumento anterior que el interés de la clase de los rentistas es opuesto al interés de la clase trabajadora. Entre más excedente vaya a la clase ociosa menos ira a los capitalistas $y$, en consecuencia, puesto que se supone que éstos invierten lo que reciben én beneficio, se adquirirá menos trabajo el siguiente año.

7.2. En el ejemplo numérico que presentamos antes vimos que, conforme a esta visión, al aumentar el beneficio aumenta el valor de cambio de las mercancias y en consecuencia aumenta la cantidad de trabajo adquirible en el siguiente periodo. Smith, pues, ve en la 
existencia de los beneficios, y por lo tanto, en la existencia de la clase capitalista, la condición necesaria para el crecimiento económico. Para Smith, es la clase capitalista el motor del crecimiento económico y del bienestar social.

\section{DISTRIBUCION Y COMPETENCIA.}

8. El precio real de las mercancias es la cantidad de trabajo que estas pueden adquirir en el mercado, y su precio nominal es esta misma cantidad valuada en dinero. Por otra parte, el precio de toda mercancía está regulado por el salario, el beneficio y la renta; esto significa que las variaciones en el precio de la mercancía se explican por las variaciones de alguno o de todos los componentes. Sin embargo, la experiencia sefiala que el precio de las mercancías puede variar por otras razones distintas y a causa de esta variación variar uno o todos los componentes. Por este motivo es necesario establecer la naturaleza distinta de estas dos tipos de variaciones, aunque vinculadas entre sí, antes de estudiar la distribución del ingreso. Este problema conduce directamente a la distinción entre precio de mercado y precio natural. Aquí se tratará la forma especlfica que adoptan los mecanismos de regulación del precio en Smith.

Smith comienza sefialando que:

"(e)n toda sociedad o comarca existe una tasa promedia o corriente de salarios y de beneficios en cada uno de los empleos distintos del trabajo y del capital...Existe también en toda sociedad o comunidad una tasa promedia 0 corriente de renta...Estos niveles corrientes 0 promedios se pueden llamar tasas naturales de los salarios, del beneficio y de la renta, en el tiempo y en el lugar en que generalmente prevalecen."(pag. 54).

Los niveles promedios o naturales de los componentes determinan el nivel natural del precio de las mercancias.

"Cuando el precio de una cosa es ni más ni menos que el suficiente para pagar la renta de la tierra, los salarios del trabajo y los beneficios del capital empleado en obtenerla, prepararla y traerla al mercado, de acuerdo con sus precios corrientes, aquella se vende por lo que se llama su precio natural."(pag. 54).

Sin embargo el precio efectivo que realmente se paga en el mercado en cada momento y lugar puede diferir del precio natural o coincidir con él. El precio natural, pues, es sólo una marca de referencia, es el precio efectivo el que se paga en el mercado, por esta razón a este último se le llama precio de mercado. Según Smith el precio de 
mercado:

"se regula por la proporción entre la cantidad (de la mercancia) que realmente se lleva al mercado y la demanda de quienes están dispuestos a pagar el precio natural del artículo"(pag. 55).

Smith llama a la cantidad de una mercancía que los compradores demandan a su precio natural, la demanda efectiva. De esta manera, si la cantidad llevada al mercado es insuficiente para cubrir la demanda efectiva, debido a la competencia entre los que desean adquirir la mercancia, el precio de mercado ascenderá sobre el precio natural; si, por el contrario, la cantidad llevada al mercado excede la demanda efectiva, el precio de mercado descenderá bajo el precio natural; solamente cuando la cantidad llevada al mercado coincide con la demanda efectiva, ambos precios seran iguales.

No obstante bajo la acción de las libres fuerzas del mercado, esta diferencia entre los precios de mercado y natural tiende continuamente a desaparecer. Cada vez que el precio de mercado se mantiene por encima del precio natural, debido a una insuficiencia de la oferta, alguno de los componentes se pagará por encima de su tasa natural.

"Si es la renla, el interés de todos los demás terratenientes hará que dediquen más tierras para el cultivo de ese fruto; si es el salario o el beneficio, el interés de los otros trabajadores y negociantes les obligará pronto a emplear más trabajo y más capital en la preparación de la mercancia y en el acarreo al mercado. La cantidad de mercaderias ofrecidas a los compradores pronto será suficiente para satisfacer la demanda efectiva, todos los componentes del precio bajarán pronto a su tasa natural, y el precio global a su precio natural."(pag. 56).

De igual forma describe el ajuste en caso de un exceso de oferta. Por esta razón, pues, el precio de mercado está siempre buscando hacia el precio natural. Como se ha descrito antes, este ajuste se desarrolla a lo largo del tiempo. El precio natural es el nivel de convergencia del precio de mercado. Smith lo atirma en los siguientes términos:

"El precio natural viene a ser, por esto, el precio central, alrededor del cual gravitan continuamente los precios de todas las mercancias. Contingencias diversas pueden a veces mantenerlos suspendidos, durante cierto tiempo, por encima o por debajo de aquel; pero, cualesquiera que sean los obstáculos que les impiden alcanzar su centro de reposo y continuación, continuamente gravitan hacia él."(pag. 57).

En el periodo largo, pues, se supone que los ajustes se han realizado, el precio de mercado ha avanzado hacia el precio natural a 
través del ajuste de la oferta hacia la demanda efectiva. En otras palabras, en la perspectiva smithiana, en el largo plazo la oferta queda determinada por la demanda; es decir, en el largo plazo, la demanda regula la producción de mercancías. No obstante, en el corto plazo, dado un nivel de demanda efectiva, cualquier deficiencia de la oferta se traducirá en incremento del precio.

Smith distingue diferencias en el ajuste de acuerdo a las caracteristicas de las distintas mercancias. Los productos de origen agricola, por ejemplo, varían en su magnitud de producción de año a año, aunque se aplique la misma cantidad de trabajo, debido entre otras cosas al comportamiento del tiempo; los productos industriales, por el contrario, se caracterizan por tener una relación trabajo-producción bastante estable. de tal forma que el producto de identicas cantidades de trabajo es siempre el mismo o aproximadamente el mismo. En consecuencia, la cantidad de producto llevada al mercado de los segundos será más facil de ajustar al nivel de demanda electiva que la de los primeros; por lo tanto, las fluctuaciones de los precios serán mucho mayores en los primeros que en los segundos, aun cuando la demanda efectiva no cambie.

"El precio de una especie de mercancias varia únicamente con las alteraciones de la demanda; el de otras, no sólo con las variaciones de la demanda, sino con arreglo a las oscilaciones, mucho mayores y más frecuentes, de las cantidades trasladadas al mercado con el fin de satisfacerla."(pag. 57).

9. El precio natural ha quedado definido en aquellas circunstancias en que la oferta se equilibra con la demanda, pero este equilibrio entre estas dos variables puede ser estático o dinámico; en el primer caso el nivel de la demanda está fijo y la oferta se ajusta a ese nivel, en el segundo, la demanda está creciendo a una tasa determinada y el equilibrio se establece ajustandose el crecimiento de la oferta a la misma tasa de crecimiento de la demanda. Esta última situación crea dificultades adicionales al concepto de tasa natural. En la determinación de la tasa natural en un análisis estático la oferta y la demanda no influyen más que para crear las variaciones del precio por encima o por debajo del nivel natural, pero no inciden en la propia determinacion de este nivel; por el contrario, como veremos a continuación, en un análisis dinámico el ritmo de expansión de la demanda influye no solamente en las variaciones del precio de mercado sino tambien en la determinación de la tasa natural.Esta precisión es importante, pues al analizar los determinantes del salario y del beneficio se tiende a confundir, como parece ser el caso de Cartelier, la determinación de la tasa natural con 
la determinación de la tasa de mercado. En este aspecto es importante el siguiente sefialamiento de Smith:

"intentaré esclarecer cuáles son las circunstancias que naturalmente determinan la tasa de los salarios, y hasta qué punto dichas circunstancias se ven afectadas por la riqueza o la pobreza, asi como por el estado progresivo, estacionario o atrasado de una sociedad."(pag. 61).

Según el párrafo anterior, sobre la tasa natural del salario influye el estado progresivo, el estacionario y el atrasado, que como veremos más adelante, son los estados de crecimiento, de estancamiento y de retroceso de la economia.

Trataremos de derivar de los conceptos vertidos por Smith acerca de la tasa natural sus implicaciones en un contexto de crecimiento. Sabemos en primer lugar que el precio de mercado crece cuando existe un exceso de demanda y desciende cuando hay una deficiencia y que estas variaciones son mayores entre mayores son estos excesos 0 insuficiencias de demanda. Establezcamos, entonces la siguiente función de variación de precios:

$$
\text { Variación de precio }=t(E D)
$$

El precio varía según haya exceso de demanda(ED), la cual puede ser positiva o negativa (insuficiencia de demanda). Supongamos equilibrio inicial entre la oferta y la demanda, de tal forma que no existe exceso de demanda, en consecuencia ED será cero y el precio de mercado no variara, por lo tanto el precio de mercado coincide con el precio natural. Supongamos ahora que la demanda de toda la economia comienza a crecer a una tasa del $10 \%$, si la oferta se queda al mismo nivel que en el periodo anterior, aparecerá un exceso de demanda el cual provocará crecimiento en los precios; esto a su vez provocará que alguno o todos los componentes del precio se paguen por encima de su tasa natural y que en consecuencia tienda a aumentar la oferta. Si la oferta responde creciendo al $5 \%$ y la demanda continúa a su ritmo anterior, el exceso de demanda continuará y en consecuencia los precios seguirán creciendo, quizas a un ritmo menor según sea el exceso de demanda. Lo cual promoverá que más recursos se dediquen a la actividad económica. Cuando idealmente el nivel de la oferta alcance el nivel de la demanda y su ritmo de crecimiento el ritmo de la demanda, el exceso de demanda desaparecerá y el precio se estabilizará a ese nuevo nivel más alto $y$, a menos que cambie el ritmo de crecimiento de la demanda de la economia y el crecimiento de la oferta, no habrá ningún motivo para que el precio cambie, por lo tanto 
se trata de un nivel natural.

Algunas consecuencias del análisis anterior para la determinación del nivel natural son las siguientes:

a. No existe un único nivel de la tasa natural, éste depende de la tasa de crecimiento de la economia. Si en el ejemplo anterior, la demanda de la economia hubiese estado creciendo más rápidamente, la tasa natural se habria ubicado a un nivel mayor. Este resultado es lógico, pues para que más recursos se volquen hacia la atención de esa demanda creciente, es necesario mayor remuneración para los factores productivos. Esta conclusión se entenderá con mayor precisión en el análisis de la tasa natural de los salarios.

b. Contrario a los resultados que se obtienen cuando el análisis es estático, la demanda si influye sobre el nivel natural de los precios. Por lo tanto el análisis de los valores naturales no se puede realizar adecuadamente si no se considera en un contexto dinámico de la demanda y la oferta. Esto es efectivamente to que hace Smith al analizar los salarios, el beneficio y la renta en los estados progresivo, estacionario y decadente de la economia.

\section{II.1. La tasa natural de salarlos.}

10. Después de definir, que por salario del trabajo se estiende aquella recompensa que se ortorga cuando el trabajador es una persona distinta del propietario del capital que emplea al obrero, Smith muestra la naturaleza de clase de la relación capital trabajo en los siguientes términos:

"Los salarios del trabajo dependen generalmente, por doquier, del contrato concertado por lo común entre estas dos partes, y cuyos intereses dificilmente coinciden. El operario desea sacar lo más posible, y los patronos dar lo menos que puedan. Los obreros están siempre dispuestos a concertarse para elevar los salarios, y los patronos, para rebajarlos."(pag. 65).

El poder de cada clase para inclinar la solución del conflicto a su favor es asimétrica:

"Sin embargo, no es difícil de prever cuál de las dos partes saldrá gananciosa en la disputa, en la mayor parte de los casos, y podrá forzar a la otra a contentarse con sus términos. Los patrones siendo menos en número, se pueden poner de acuerdo más facilmente, ademas de que las leyes autorizan sus asociaciones 0 , por lo menos, no las prohiben, mientras que, en el caso de los trabajadores, las desautorizan...En disputas de esa indole los patrones pueden resistir mucho más tiempo. 
Un propietario, un colono, un fabricante o un comerciante, aun cuando no empleen un solo trabajador, pueden generalmente vivir un año o dos, disponiendo del capital previamente adquirido. La mayor parte de los trabajadores no podrán subsistir una semana, pocos resistirán un mes, y apenas habrá uno que soporte un año sin empleo. A largo plazo, tanto el trabajador como el patrono se necesitan mútuamente; pero con distinta urgencia."(pag. 65).

El dramatismo de la lucha de clase lo expresa Smith de la siguiente manera:

"En su afán de lograr una resolución pronta, los obreros promueven alborotos y, a veces, recurren a la violencia y al ultraje más ofensivos. En su desesperación, proceden los trabajadores con el trenesí propio de los desesperedos, y tienen que optar entre morir de hambre 0 atemorizar a los patronos, para que éstos accedan inmediatamente a sus pretenciones. Los patronos, en tales circunstancias, protestan en el mismo tono, y jamás dejan de reclamar la asistencia de la autoridades civiles y la aplicación inflexible de las rigurosas leyes que han sido promulgadas contra criados, trabajadores y jornaleros."(pag. 66).

Los párrafos anteriores muestran la distancia que hay entre las visiones de los economistas modernos que dicen descender directamente de Adam Smith, acerca de la armonia de clases o de la inexistencia de las clases, y la visión del maestro mismo. La relevancia de la descripción para los tiempos actuales es incuestionable.

11. Después de mostrar la naturaleza conflictiva de la relación entre el capital y el trabajo, Smith pasa a desarrollar su teoría de la determinación salarial. Según ésta el salario natural queda determinado por el crecimiento de la demanda y ésta por el incremento de los capitales que se destinan al pago de dichas remuneraciones. La teoria de Smith está compuesta de dos hipótesis: la primera, una hipótesis del comportamiento de los salarios ante los desequilibrios de la oferta y la demanda de trabajadores; y la segunda, una hipótesis del ajuste de la población ante los movimientos de los salarios. Esta última será objeto de un análisis detallado, pues ha sido motivo de fuertes críticas en el pasado.

Supongamos inicialmente una sociedad cuya riqueza ha permanecido durante largo tiempo al mismo nivel, es decir en un estado estacionario. En este estado de cosas,

"Apenas habrá escacez de mano de obra, y los patronos no se verán obligados a competir para conseguirla. Por el contrario, la mano de obra excederá, en este caso, naturalmente, las oportunidades de 
ocupación. Habrá una constante escasez de empleos y los trabajadores se verán obligados a competir entre sí, para conseguir trabajo. Si los salarios del trabajo, en un país de estas condiciones, llegaran a ser más que suficientes para mantener a los trabajadores y brindarles la oportunidad de criar una familia, su misma competencia y el interés de los amos los reduciria muy pronto al nivel más bajo compatible con la existencia humana."(pag. 70).

China es desde el punto de vista de Smith la evidencia histórica del tipo de sociedad que se acaba de suponer. En este pais:

"Las relaciones de todos los viajeros, a pesar de discrepar en otros muchos respectos, convienen en lo bajos que son los salarios del trabajo y en las dificultades con que tropiezan los obreros para poder mantener una familia."(pag. 70).

Sin embargo el nivel de salario que se establece más los esfuerzos de los trabajadores por sobrevivir hacen que la oferta disponible de trabajadores no disminuya del nivel necesario para la demanda existente:

En China,"(l)a clase inferior del pueblo trabajador hace los mayores esfurzos para que, de un modo $u$ otro, continúe propagandose la especie y no disminuya su número, a pesar de la escasez de sus mantenimientos."(pag. 71).

El salario que se establece en estas condiciones de equilibrio de oferta y demanda es el salario natural que corresponde a un estado estacionario. En estas condiciones, el salario deberia alcanzar para asegurar la reproducción de la especie al mismo nivel, debería alcanzar para que cada matrimonio lograra procrear dos hijos que les sucedieran como fuerza de trabajo. A. Smith sefiala que, desde un punto de vista humano, éste sería el nivel mínimo:

"por bajo del cual parece imposible que baje, a lo largo del tiempo, el salario corriente de las ocupaciones de inferior categoria."(pag. 66).

Debe observarse que a Smith solamente le parece imposible que baje, pues esto es lo que ocurre en el estado decadente de la sociedad, cuando la economía está en retroceso, cuando la demanda de trabajadores diminuye año tras ano. En estas circunstancias:

"(c)ada año iría siendo menos que la anterior la demanda de criados y trabajadores en toda clase de empleos...La clase más baja...., registraría una competencia tan grande, por parte de quienes buscan empleo, que los salarios del trabajo se reducirian al nivel de la más miserable y escasa subsistencia del obrero.....; correrian el riesgo de 
morir de hambre...La miseria, el hambre, la mortandad prevalecerían muy pronto en esta clase desdichada, y de ella el contagio pasaria a las superiores, hasta que el número de habitantes del pais quedase reducido a los que fácilmente pueden sustentar el ingreso y el capital que todavia quedasen"(pags. 71, 72).

Cuando la demanda de trabajadores está en retroceso, el salario cae por debajo de aquel nivel minimo que sefialaba Smith; considerando el proceso de muerte que esta situación supone, a fin de que la oferta de trabajadores también entre en retroceso para ajustarla a la demanda, es razonable que Smith haya considerado aquel nivel como el más bajo desde un punto de vista humano. Una situación dramática como la antes supuesta es la que Smith observa en su época en algunos países:

"Este es aproximadamente el estado actual de Bengala y de algunos otros establecimientos ingleses en las Indias Orientales....(alli) el hecho de que mueran de hambre y miseria de trescientas a cuatrocientas mil personas en un año, es señal evidente de que los fondos destinados a mantener al pobre trabajador se halian próximos a agotarse."

Como se vé la demanda de trabajadores, aun cuando está decreciendo, regula su oferta; asi, en estas condiciones se establece una tasa natural de salarios que regula el movimiento de los salarios de mercado, una tasa natural que está por debajo de la tasa natural del estado estacionario.

Lo contrario ocurre cuando la demanda de trabajadores está creciendo:

"Cuando en un pais aumenta continuamente la demanda de aquellas personas que viven de su salario...(I)a escasez de mano de obra origina una competencia entre los patronos, y éstos porfian entre si para contratar a aquéllos, con lo que voluntariamente se rompe la natural coalición de los patronos para no subir los jornales."(pag. 67).

En consecuencia, argumenta Smith, 10 que motiva el alza de salarios, no es la magnitud real de la riqueza de la nación, sino su continuo incremento.

El argumento de Smith continua mostrando que de continuar el crecimiento de la demanda la olerta de trabajo crecerá hasta alcanzar el ritmo de crecimiento de la demanda. El argumento que presenta merece atención detallada.

La pobreza está asociada a dos consecuencias, la fecundidad y la 
deficiencia en la capacidad de criar los hijos; lo contrario ocurre con la riqueza.

"Una montaniesa, aunque medio muerta de hambre, tiene por lo común, más de veinte hijos, mientras que una dama criada en la abundancia es, a menudo, incapaz de tener más de uno, y se agota cuando da a luz dos o tres....Se oye decir con trecuencia que en las tierras altas de Escocia la madre que ha tenido veinte hijos apenas conserva dos....En ciertos lugares mueren, (los ninos), por lo regular, antes de la edad de cuatro años, en otros, antes de los siete, y, en los más, sin llegar a los diez."(pag. 77).

Estas observaciones de Smith han sido recogidas de la realidad histórica de su tiempo, es decir, de la época del despegue del capitalismo. Una época que, además, se caracterizaba por convertir a los niños de corta edad en fuerza de trabajo.De estas condiciones se desprende facilmente que el aumento del ingreso de-las clases más pobres de la sociedad, a través del incremento de los salarios, traería como resultado la mejor atención de los hijos y mejores condiciones para criarlos, reduciendo en consecuencia la tasa de mortalıdad infantil, ampliando en un corto tiempo la población. Si se toma en cuenta que en la época que Smith estaba escribiendo, los niños de corta edad constituían fuerza de trabajo es natural que viera en la reducción de la tasa de mortalidad infantil un mecanismo de ampliación relativamente rápido de la fuerza de trabajo.

Así, pues, Smith concluye su argumento indicando que cuando la demanda de trabajo crece más rápidamente que su disponibilidad, el alza de salarios que provoca tal situación se encarga de crear las condiciones para que la oferta de trabajo crezca al ritmo de aquella. Cuando la oferta de trabajo ha alcanzado la tasa necesaria exigida por la demanda, el salario deja de crecer; este nuevo nivel alcanzado por el salario es una tasa natural. Si circunstancias accidentales hicieran aumentar o disminuir la oferta de trabajo, el salario de mercado disminuirla o aumentaría con respecto a esta tasa natural, pero el mecanismo poblacional antes descrito ajustaria el salario de mercado después de un periodo de tiempo al nivel natural arriba indicado. El desajuste de la oferta seria únicamente temporal.

Dos conclusiones se derivan del razonamiento de Smith. La primera es que la demanda de trabajadores crea su propia oferta:

"Asi es como la demanda de hombres, al igual de lo que ocurre con las demás mercancías, regula de una manera necesaria la producción de la especie, acelerandola cuando va lenta y f́renandola cuando se aviva demasiado."(pag. 78). 
La segunda, que a cada tasa de crecimiento de la demanda de trabajadores corresponde una tasa natural de salarios. Esta última conclusión es importante de destacarla pues es diferente a la obtenida por Cartelier, quien señala que en Smith sólo existe una tasa natural de salarios, aquella correpondiente al estado estacionario. Si ésta fuera la única tasa natural, entonces el crecimiento de la demanda alzaria el salario de mercado por encima del nivel natural sólo temporalmente hasta que la oferta de trabajo creciera al mismo ritmo de la demanda, una vez esto se lograra el salario bajarla nuevamente a su nivel natural correspondiente al estado estacionario, aun cuando la economía estuviera en crecimiento equilibrado. Si asi ocurriera, entonces, la población comenzaria a decrecer al nivel del estado estacionario y si la demanda sigue creciendo al mismo ritmo que hemos supuesto se crearia un desequilibrio que impulsaría los salarios nuevamente hacia arriba, to cual es contrario al concepto de tasa natural de equilibrio.

12. Según el estado de la demanda de trabajadores así será la cantidad de bienes que podrá obtener el obrero a cambio de su trabajo; cuando la demanda de trabajadores se acelera el trabajador deberá poder adquirir más bienes a fin de que en verdad pueda resultar un efecto positivo sobre la población a través de la mejora en sus condiciones de vida. Por tanto, el precio monetario de las provisiones regulará el precio nominal del trabajo, es decir, el salario monetario. Si, por ejemplo, la demanda de trabajadores se acelera y el precio de las provisiones disminuye, el salario monetario podría permanecer sin ninguna variación, si es que la segunda causa de variación de los salarios compensa suficientemente la segunda.

"El precio en dinero de este último (del trabajo) se regula necesariamente por dos circunstancias: la demanda de trabajo y el precio de las cosas que son necesarias y útiles para la vida."(pag. 83).

Un corolario importante de la teoría del valor y de los salarios es que el alza del precio de las provisiones aumentará el precio nominal del trabajo y éste se trasladará al precio de todas las demás mercancias. Esta es una consecuencia lógica y Smith la establece en los siguientes términos:

“(S)abido es, en efecto, que el precio del grano en dinero regula el de todos los demás artículos de producción nacional...Regula el precio nominal del trabajo, el cual ha de ser de tal naturaleza que faculte al trabajador para comprar una cantidad de trigo suficiente para atender a su mantenimiento y al de su familia, en la forma abundosa, moderada o escasa con que las circunstancias del estado progresivo, estacionario o decadente del pais obliguen a mantenerlos a los patronos... Regulando 
el precio de los salarios del trabajo, ha de regular el de las artes manufactureras y de la industria."(pag. 452).

Este corolario será retomado en un trabajo posterior sobre el pensamiento económico de David Ricardo que se encuentra en proceso.

\section{II.2. La tasa natural de beneficlo.}

13. Al igual que con la tasa natural de salarios, Smith hace depender la determinación de la tasa natural de beneficio de la acumulación de capital. Esta determinación, sin embargo, está provista de algunas características especiales a las cuales habrá que dedicarles atención especial.

Smith comienza afirmando que son las mismas causas de la variación de los salarios las que hacen variar los beneficios; éstas son las mismas que vuelven una sociedad progresiva, estacionaria o decadente, es decir, la acumulaión de capital, sin embargo, estas causas operan de manera muy distinta en un caso y en otro. Una de las maneras distintas de operar es que produce efectos inversos:

"El aumento de capital, que hace subir los salarios, propende a disminuir el beneficio."(pag. 85).

Y esto es asi, porque la acumulación de capital desencadena la competencia por los mercados estre los capitalistas. El argumento de Smith se desarrolla en dos pasos. Al nivel microeconómico el autor afirma:

"Cuando los capitales de muchos comerciantes ricos se invierten en el mismo negocio, la natural competencia que se hacen entre ellos tiende a reducir su beneficio."(pag. 85).

Esto es correcto, pues al aumentar el capital en una rama de la producción, su oferta de productos excederá a la demanda efectiva correspondiente, de donde resultará una caída del precio de mercado. Es esta caida del precio la que hace disminuir la tasa de beneficio de mercado por debajo de su nivel natural. De esta conclusión, Smith da el salto al nivel macroeconómico, concluyendo finalmente que:

"cuando tiene lugar un aumento del capital en las diferentes actividades que se desempenan en la respectiva sociedad la misma competencia producirá efectos similares en todas ellas."(pag. 85).

Smith es preciso en los mecanismos que a nivel macroeconómico harán que la acumulación de capital disminuya la tasa de beneficio en el capítulo IV del Libro Segundo : 
"A tenor que aumentan los capitales en un país, los beneficios resultantes de su empleo disminuyen necesariamente. Cada vez se hace más dificil encontrar un procedimiento para colocar en forma rentable el nuevo capital en la nación. Surge por tal causa, la competencia entre los diferentes capitales, porque los dueños de éstos buscan aquella colocación que ya está ocupada por otros. Las más de las veces pretenden arrebatar a los demás el empleo, ofreciendo condiciones más ventajosas. No sólo es necesario vender algo más barato dentro del ramo, sino que, en ocasiones, para lograr la venta, se compra a veces más caro."(pag. 319,320).

La conclusión anterior será válida solamente si se supone que la demanda de bienes está fija y que la acumulación de capital se desarrolla en las mismas ramas ya existentes. Smith es conocedor de este supuesto pues en otra parte reconoce las implicaciones de su no cumplimiento:

"La incorporación de un nuevo territorio o de nuevas ramas del comercio puede en algunas ocasiones elevar los beneficios del capital, y con éstos el interés del dinero, aun en un país que progresa rápidamente en la adquisición de riqueza... Parte del capital que antes se empleaba en otros negocios se retira de ellos necesariamente, para invertirse en ciertas operaciones nuevas, que son más ventajosas. La competencia, por lo tanto, disminuye en los negocios de antiguo arraigo. El mercado se halla ahora menos surtido de ciertas clases de mercancias. Sube más o menos el precio de éstas, proporcionando un mayor beneficio a los que trafican en ellas.."(pag. 91).

14. Este paso de una conclusión microeconómico a su generalización macroeconómica no considera todas las implicaciones de las interrelaciones de un sistema económico completo y en este sentido la interpretación que Smith hace de los determinantes de la tasa de beneficio es incompleta. Aplicando su lógica se puede establecer de qué manera se determina la tasa natural de beneficio. Supongase que la sociedad se encuentra en un estado estacionario al cual corresponden determinadas tasas naturales de salario y de beneficio. Supongase que en estas condiciones se da una cierta acumulación de capital en una rama particular. El resultado será, como ya se argumentó, un exceso de oferta del bien producido en esta rama respecto de la demanda efectiva a su precio natural. Su precio de mercado descenderá por debajo del nivel natural con lo cual la tasa de beneficio de mercado en esta rama descenderá también por debajo de la natural. No se puede esperar un descenso del salario de mercado, pues por la acumulación de capital lo que se puede esperar, por el contrario, es un 
aumento de la demanda de trabajadores que tendería a aumentar los salarios. Supongase por el momento sólo el efecto sobre los beneficios. El capital tenderá a alejarse de esta rama de baja tasa de beneficio hacia las restantes ramas. Como consecuencia de este movimiento el precio del bien producido por la rama donde originalmente se dió la acumulación de capital tenderá a aumentar y los precios de los restantes bienes tenderán a disminuir, obligando a que la tasa de beneficio tienda a subir allá y a disminuir aqul. El desplazamiento de capital cesará hasta que ambas tasas de beneficio se hallan igualado. Obviamente este nivel de tasa de beneficio de equilibrio es una tasa natural inferior a la tasa inicial; la razón es que existe un capital al final del proceso mayor que el original. En esta posición final de equilibrio también los precios naturales de los bienes serán diferentes y, por lo tanto, también lo serán las demandas efectivas correspondientes.

Qué pasará si acaso la acumulación de capital continúa periodo tras periodo? Si ésta continua dandose en las mismas ramas, la tasa de beneficio de mercado se estará ajustando hacia una tasa natural que no está fija, sino desplazandose continuamente hacia bajo. Cuando la acumulación de capital cese, la tasa natural de beneficio dejará de descender y la de mercado buscará hacia el nivel alcanzado por aquella.

15. Pero, la continuación de la acumulación de capital crea un crecimiento sostenido de la demanda de trabajo, con lo cual después del ajuste en el ritmo de crecimiento de la población establece un nivel de tasa natural de salarios más elevada. Por el lado del beneficio, sin embargo, el mismo ritmo sostenido de acumulación de capital crea un descenso continuado de la tasa natural de beneficio, la cual sólo alcanzará un nivel fijo hasta que cese la acumulación.

En un estado progresivo, pues, la tasa natural de salarios alcanza un nivel más elevado de acuerdo al grado de acumulación que sostiene este estado, y la tasa natural de beneficios se encuentra en un proceso continuado de descenso. El movimiento de los precios naturales dependerá del efecto combinado del alza de la tasa natural de salarios y del movimiento descendente de la tasa de beneficio.

16. Aun es necesario considerar que la demanda efectiva no puede considerarse modificada únicamente por el movimiento de los precios naturales en un proceso de acumulación sostenida, pues el crecimiento de la población que provoca la acumulación, de acuerdo a la lógica de Smith, equivale a un crecimiento de la demanda efectiva, ya que aquella es el resultado del aumento de los ingresos de las clases más pobres por causa de los aumentos de salarios ante el aumento de la demanda 
de trabajadores.

La caída de los precios frente a la acumulación es contrarrestada en alguna medida tanto por el crecimiento de la demanda efectiva de medios de producción, resultado directo de la acumulación de capital; como por el crecimiento de la demanda efectiva de bienes de consumo para los trabajadores, por las razones que arriba se expusieron.

17. Debe observarse en la determinación de las tasas naturales de salarios y beneficios algunas diferencias adicionales en el papel que juega sobre la última el comportamiento de los precios. En la determinación de la tasa natural del salario el movimiento de los precios de los bienes no influye de ninguna manera, pues el ajuste de la oferta de trabajo hacia su demanda se da a través del movimiento directo del salario; mientras que, por el contrario, en la determinación de la tasa natural del beneficio es el nivel de precios el que se ajusta para alcanzar la tasa de equilibrio del beneficio. Pero de esto no debe concluirse como muchas veces se ha hecho que en la determinación de la tasa de beneficio existe circularidad, pues lo que realmente existe es simultaneidad. Se ha visto que existe una tasa natural de beneficio compatible con niveles determinados de capital y de demanda global, si el acervo de capital aumentara, ceteris paribus, la tasa natural de beneficio tendria que disminuir, y los precios naturales de todos los bienes tendrian que variar, a través de los desplazamientos del capital entre sectores, a fin de que se imponga la nueva tasa natural de beneficio. Asi, pues, la tasa natural de beneficio queda determinada, en el sistema smithiano, por la proporcionalidad entre el stock acumulado de capital y la demanda global, y los precios finales se ajustan para contener aquella cantidad como un componente.

18. Si hay circularidad en la determinación de la tasa de beneficio en cuanto que su determinación supone conocido el valor del acervo de capital empleado, pero la cantidad de trabajo que este acervo puede adquirir, o lo que es lo mismo su valor de cambio, no puede determinarse sin antes haber determinado la tasa de beneficio, pues ésta es una de las componentes del valor. En sintesis, pues, el problema de circularidad consiste en que para determinar la tasa de beneficio es necesario haber determiado previamente los precios, pero estos no pueden determinarse si no se conoce la tasa de beneficio, pero este es un problema que se tratará con profundidad en otro trabajo.

Basten los análisis de los salarios y del beneficio para entender los argumentos de Smith y sus implicaciones para su teoria del valor y la distribución. 
El de Smith fue el primer esfuerzo por sistematizar una teoria del valor y la distribución, esfuerzo que sirvió de base, incluido su metodologia de los niveles naturales, para los desarrollos posteriores del tema desde Ricardo hasta los tiempos actuales.

Smith no andaba buscando la sustancia del valor a través del trabajo, sino solamente el patrón de medida más adecuado de los precios, y éste lo encontró en el trabajo adquirible. De esto resulta que, contrario a lo que se ha argumentado, en Smith no existen dos teorias del valor, una que se basa en que el valor es la cantidad de trabajo empleado en la producción de un bien, y otra, que sustituye a la primera, en la cual el valor queda determinado por la cantidad de trabajo adquirible. Por el contrario, se puede encontrar que para él el valor queda determinado a lo largo de toda su obra por la cantidad de trabajo adquirible $y$, por lo tanto, su teoria del valor es única.

A diferencia de los autores posteriores, exceptuando a Marx, Smith trató de desarrollar su análisis en un contexto dinámico, lo cual le adiciona connotaciones especiales a la determinación de los niveles naturales de las variables. Como se desprende de los argumentos presentados, también contrario a algunas afirmaciones de otros autores, no existe una unica tasa natural de precios, de salarios y de beneficios en un contexto dinámico, sino que aquellas quedan determinadas por el ritmo de expansión de la demanda en la economía, es decir por el estado progresivo, estacionario o decadente de la sociedad.

No hay duda que los argumentos a nivel macroeconómico de la determinacion de la tasa natural de beneficios de Smith son insuficientes, pues, dada la complejidad de su modelo, para captar adecuadamente todas sus implicaciones requeriria de un desarrollo más riguroso, es decir de su formalización matemática.

\section{REFERENCIAS}

Smith, Adam (1982)."Investigación sobre la Naturaleza y Causas de la Riqueza de la Naciones". FCE. México.

Cartelier, Jean (1981). "Excedente y Reproducción. La Formación de la Economía Política y Clásican. FCE. México. 